Relationship of Neuropsychiatric Symptoms with Falls in Alzheimer's Disease : Does Exercise Modify the Risk?

\title{
Roitto, Hanna-Maria
}

2018-12

Roitto , H-M , Kautiainen , H , Öhman , H , Savikko , N , Strandberg , T E , Raivio , M , Laakkonen, M-L \& Pitkälä , K H 2018 , ' Relationship of Neuropsychiatric Symptoms with Falls in Alzheimer's Disease : Does Exercise Modify the Risk? ' , Journal of the American Geriatrics Society, vol. 66 , no. 12 , pp. 2377-2381 . https://doi.org/10.1111/jgs.15614

http://hdl.handle.net/10138/308826

https://doi.org/10.1111/jgs.15614

publishedVersion

Downloaded from Helda, University of Helsinki institutional repository.

This is an electronic reprint of the original article.

This reprint may differ from the original in pagination and typographic detail.

Please cite the original version. 


\title{
Relationship of Neuropsychiatric Symptoms with Falls in Alzheimer's Disease - Does Exercise Modify the Risk?
}

\author{
Hanna-Maria Roitto, MD, ${ }^{* \dagger}$ Hannu Kautiainen, PhD, biostatistician, ${ }^{*}$ Hannareeta Öhman, MD, $* \dot{\dagger}$ \\ Niina Savikko, RN, PhD, *\$ Timo E. Strandberg, MD, PhD, ${ }^{\mathbb{T} / \| *}$ Minna Raivio, MD, PhD, * \\ Marja-Liisa Laakkonen, MD, PhD, ${ }^{* \dagger}$ and Kaisu H. Pitkälä, MD, PhD ${ }^{* *}$
}

OBJECTIVES: To explore how neuropsychiatric symptoms (NPS) are associated with number of falls and how exercise modifies the risk of falling in community-dwelling people with Alzheimer's disease (AD) and NPS.

DESIGN: Secondary analysis of a randomized controlled trial.

SETTING: Community.

PARTICIPANTS: Community-dwelling individuals with AD $(\mathrm{N}=210)$ who completed the Neuropsychiatric Inventory (NPI) $(\mathrm{N}=179)$.

INTERVENTION: Participants were randomized into 3 groups: group-based exercise (4-hour sessions with approximately 1 hour of training) and tailored home-based exercise (1 hour of training) twice a week for 1 year and a control group receiving usual community care. In this secondary analysis, we merged the home-based and groupbased exercise groups and compared this group with the control group.

MEASUREMENTS: NPS were measured using the NPI at baseline, and spousal caregivers recorded falls in daily fall diaries during 1 year of follow-up.

RESULTS: The number of falls increased linearly with NPI score in the control group. Fall rates were 1.48 (95\% confidence interval $(\mathrm{CI})=1.26-1.73)$ per person-year in the intervention group and $2.87(95 \% \mathrm{CI}=2.43-3.35)$ in the control group. Adjusted for age, sex, Mini-Mental State Examination

From the *Department of General Practice, University of Helsinki, Helsinki, Finland; ${ }^{\dagger}$ Department of Social Services and Health Care, Helsinki Hospital, Helsinki, Finland; ${ }^{+}$Unit of Primary Health Care, Helsinki University Hospital, Helsinki, Finland; ${ }^{\S}$ Home Care, City of Espoo, Espoo, Finland; "Department of Medicine, University of Helsinki, Helsinki, Finland; "Geriatric Clinic, Helsinki University Hospital, Helsinki, Finland; and the ${ }^{* *}$ Center for Life Course Health Research, University of Oulu, Oulu, Finland.

Address correspondence to Hanna-Maria Roitto, MD, Department of Social Services and Health Care, Geriatric Clinic, PO Box 6600, City of Helsinki FI-00099, Finland. E-mail: hanna-maria.roitto@hel.fi or roittohannamaria@gmail.com

DOI: $10.1111 /$ jgs.15614
(MMSE) score, and Short Physical Performance Battery (SPPB) score, incidence rate ratio (IRR) was $0.48(95 \%$ $\mathrm{Cl}=0.39-0.60, \mathrm{p}<.001)$. Main effects for fall rate were significant for group $(\mathrm{p}<.001)$ and NPI total $(\mathrm{p}<.02)$; the interaction effect was also significant $(\mathrm{p}=.009)$ (adjusted for sex, age, MMSE score, SPPB score, and psychotropic medication use).

CONCLUSION: Exercise may decrease the risk of falling in community-dwelling individuals with AD and NPS. Future exercise trials should confirm this finding in participants with significant NPS.

TRIAL REGISTRATION: ACTRN12608000037303. J Am Geriatr Soc 66:2377-2381, 2018.

Key words: neuropsychiatric symptoms; NPI; Alzheimer's disease; exercise; falls

A lzheimer's disease $(\mathrm{AD})$ is characterized by cognitive and functional decline ${ }^{1}$ and is associated with neuropsychiatric symptoms (NPS) and falls. ${ }^{2,3}$ Up to $90 \%$ of individuals with $\mathrm{AD}$ will experience at least 1 significant NPS during the course of the disease. ${ }^{4}$ NPS seem to be highly persistent, and clinically significant symptoms may indicate poor prognosis, caregiver burden, and high care costs. ${ }^{4}$

People with dementia have a significantly higher risk of falling than those without. ${ }^{2}$ The risk is twice as high community-dwelling people with dementia than in those without dementia. ${ }^{5}$ Approximately $60 \%$ of older persons with dementia or cognitive impairment fall annually., Studies have suggested that NPS predict falls. ${ }^{8-10}$

Exercise reduces the number of falls in older adults with dementia. ${ }^{11}$ The Finnish Alzheimer Disease Exercise (FINALEX) trial showed that a long-term intensive exercise intervention decreased the number of falls in communitydwelling people with AD. ${ }^{12}$ Few studies have explored the association between NPS and falls. Even less is known about how exercise modifies the risk of falls in people with dementia and NPS. The aim of this study was to examine 
the relationship between NPS and falls and whether a longterm exercise intervention would reduce the risk of falling in community-dwelling people with AD and NPS.

\section{METHODS}

This report is a secondary analysis of a randomized intervention trial investigating the effects of an exercise program on older adults with AD (FINALEX). The study included individuals in all stages of AD with NPS. The design, baseline findings, feasibility, and endpoints of the trial have been presented previously. ${ }^{2,13}$ The original FINALEX study examined and compared home-based exercise and groupbased exercise groups, with the control group receiving regular community care. Physiotherapists supervised both intervention arms, which included similar components such as balance, endurance, strength, and multitask training. The 1-hour supervised training took place twice a week over 1 year in both groups. For this secondary analysis, we merged the 2 exercise groups into a single intervention group that we compared with the control group to increase the statistical power of the study.

\section{Participants}

The Social Insurance Institution of Finland recruited community-dwelling people with AD living in the cities of Helsinki, Vantaa, and Espoo to this study based on its drug reimbursement register. For an individual to be included in this register, a neurologist or a geriatrician must have diagnosed him or her with AD based on the National Institute of Neurological and Communicative Disorders and Stroke and the Alzheimer's Disease and Related Disorders Association Alzheimer's criteria. A letter offering the possibility of participating in the exercise trial was mailed to all 1,264 of these individuals. Those who expressed an interest in participating $(\mathrm{n}=497)$ received a postal questionnaire asking for information on inclusion criteria: established $\mathrm{AD}$, aged 65 and older, living with a spouse at home, no diagnosed terminal disease, and walking independently with or without a mobility aid. Participants were also required to have at least 1 of the following signs of frailty: 1 or more falls in the past year, a decrease walking speed, and unintentional weight loss. After receiving the completed questionnaire, the study nurse conducted a telephone interview with the spousal caregiver to ensure that all inclusion criteria were met. Two hundred ten participants met the inclusion criteria and were enrolled in the study. In this substudy, we included all participants $(\mathrm{N}=179)$ whose spousal caregiver completed the Neuropsychiatric Inventory $(\mathrm{NPI})^{14}$ information in the assessments.

\section{Measures}

A trained nurse and a physiotherapist assessed all participants and interviewed their spousal caregivers. Data on demographic factors were collected at baseline (age, sex, education, medication, comorbidities, and severity of AD based on the Mini-Mental State Examination (MMSE) ${ }^{15}$ and Clinical Dementia Rating (CDR). ${ }^{16}$ Functional status was evaluated using the Functional Independence Measure (FIM). ${ }^{17}$ Mobility was assessed using the Short
Physical Performance Battery (SPPB). ${ }^{18}$ Nutritional state was assessed and graded using the Mini Nutritional Assessment (MNA). ${ }^{19}$ Spouses gave information on falls in the individuals with $\mathrm{AD}$ during the year preceding the trial. Information on participant diagnoses and medications was retrieved from their medical records. The Charlson Comorbidity Index was calculated, as described elsewhere. ${ }^{20}$ Medications were classified using the Anatomical Therapeutic Chemical (ATC) classification system (WHO, 2015). We included acetylcholinesterase inhibitors (N06DA) and memantine (N06DX01) as AD medications. Psychotropic medications included antipsychotics (N05A), antidepressants (N06A), anxiolytics (N05B), and hypnotics and sedatives (N05C).

To evaluate NPS, spouses were interviewed using the NPI,${ }^{14}$ which asks about 12 common dementia NPS (delusions, hallucinations, agitation, dysphoria, anxiety, apathy, irritability, euphoria, disinhibition, aberrant motor behavior, sleep disturbances, eating abnormalities). For each symptom, severity is multiplied by frequency, and the sum score provides the total NPI score (range 0-144). Subsyndromes of hyperactivity, mood and apathy, and psychosis were calculated separately, as described previously. ${ }^{21}$ Depression was evaluated using the Cornell Depression Scale. ${ }^{22}$ Participants' spouses recorded falls during the 1 year of follow-up in daily fall diaries.

\section{Intervention}

A detailed description of the intervention has been provided previously. ${ }^{23}$ Before the intervention, a geriatrician assessed each participant's health status to ensure the participant's safety. The intervention groups exercised under the supervision of a physiotherapist for 1 hour twice a week over 1 year in their own home or at the gym. The exercise sessions comprised strength, balance, endurance, and multitask training, including training on a restorator bicycle, Nordic walking, stair climbing, picking up items from the floor, and talking while walking. The control group received normal community care (rehabilitation in the public healthcare system, including physiotherapy if needed).

\section{Statistics}

Sociodemographic, clinical, and drug treatment data of the groups were compared using the t-test for normally distributed continuous variables, bootstrapped type t-test for non-normally distributed continuous variables, Wilcoxon rank-sum test for differences in NPI, chi-square test for categorical variables, and Fisher-Freeman-Halton test for differences in CDR and MNA. The number and incidence rate of falls were calculated assuming a Poisson distribution. Adjusted incidence rate and incidence rate ratio (IRR) were calculated using a Poisson regression model that included sex, age, MMSE score, and SPPB total score as covariates. The Poisson regression was tested using goodness of fit of the model, and the assumption of overdispersion in the Poisson model was tested using the Lagrange multiplier test. The normality of variables was evaluated using the Shapiro-Wilk 
W test. All analyses were performed using Stata version 15.0 (Stata Corp., College Station, TX).

\section{Statement of Ethics}

The Helsinki University Central Hospital ethics committee approved the study protocol. Informed consent was obtained from each participant or, in case of significant cognitive decline (CDR 2 or 3), from the closest proxy.

\section{RESULTS}

There were no significant differences between the study groups in baseline characteristics (Table 1). Mean participant age was 78, and two-thirds were men. Two-thirds had advanced dementia (CDR 2-3), and mean MMSE score was 18. Most participants were taking AD medication, and one-third were taking a psychotropic medication. Participants had a mean of 2.5 comorbidities, and mean FIM score was 89 (range 18-126). Only one-quarter were well nourished according to MNA. Thirty-nine percent had fallen during the year preceding the intervention. The participants had few and mild NPS. Mean participant NPI score was 14.0.

\section{Interaction Effects of Exercise Intervention and NPI Score on Fall Rate}

Falls had a clear relationship with NPI score in the control group. The incidence of falls increased linearly with NPI score in the control group, whereas the exercise intervention group showed no such relationship. Fall rate was 1.48 (95\% CI $=1.26-1.73)$ per person-years in the intervention group and 2.87 (95\% CI=2.43-3.35) in the control group. Adjusted for age, sex, MMSE score, and SPPB score, IRR was $0.48,(95 \% \mathrm{CI}=0.39-0.60),(\mathrm{p}<.001)$ for the intervention group compared with the control group. Figure 1 shows the interaction in fall rate between groups and NPI baseline total score. The main effects for fall rate were significant for group $(\mathrm{p}<.001)$ and NPI total $(\mathrm{p}<.02)$; the

Table 1. Baseline Characteristics of Exercise Intervention and Control Groups

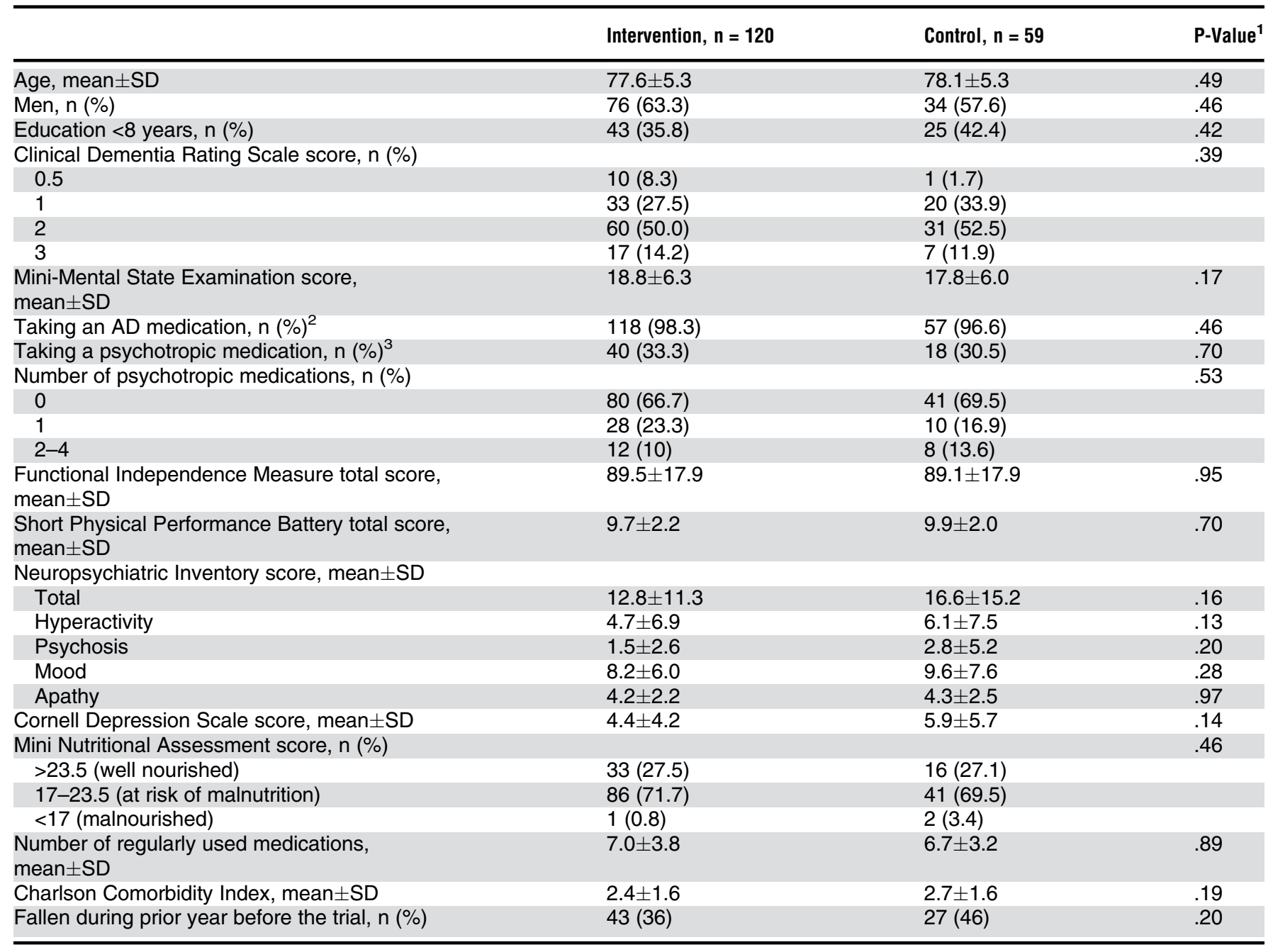

${ }^{1}$ Differences between the groups were tested using the t-test, bootstrapped type t-test, Wilcoxon rank-sum test, chi-square test, or Fisher-Freeman-Halton test.

${ }^{2}$ Cholinesterase inhibitor (N06DA), memantine (N06DX01).

${ }^{3}$ Antipsychotic (N05A), antidepressant (N06A), anxiolytic (N05B), hypnotic and sedative (N05C).

$\mathrm{SD}=$ standard deviation. 


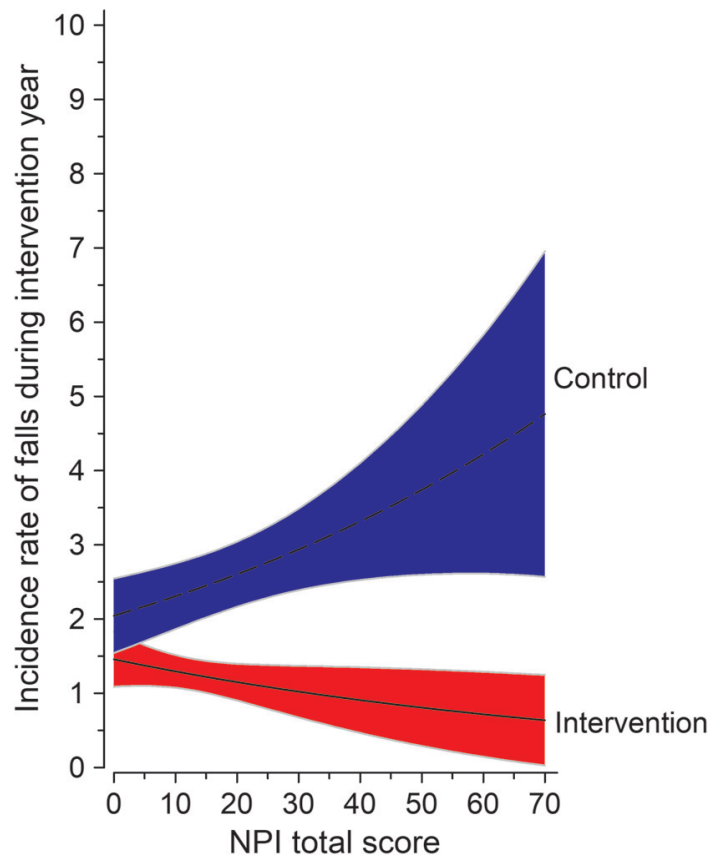

Figure 1. Incidence rate of falls in the exercise intervention and control groups according to Neuropsychiatric Inventory (NPI) score.

interaction effect was also significant $(\mathrm{p}=.009)$ (adjusted for sex, age, MMSE score, SPPB score, and psychotropic medication use).

\section{DISCUSSION}

These results show that a long-term exercise intervention reduces the risk of falling related to NPS in individuals with AD. The risk of falling increased linearly with NPI score in the control group, but exercise eliminated this risk in the intervention group. To our knowledge, no other study has examined whether exercise modifies the risk of falling related to NPS in community-dwelling people with AD.

This study has several strengths. Every participant had a confirmed diagnosis of AD. The exercise interventions were simple and clearly described. ${ }^{12}$ Adherence to the intervention was high, and the outcome measures were valid. Participants' spouses recorded falls in daily fall diaries. This method is highly sensitive to accurate recording of falls. ${ }^{24}$ The study also has some limitations that should be considered when interpreting the results. All participants were community-dwelling Caucasians living with their spousal caregivers. Because older couples were recruited, and men are more likely to have a surviving spouse, two-thirds of the participants with $\mathrm{AD}$ were men. Generalizing these results to other groups of individuals $\mathrm{AD}$ should be done with caution. Nevertheless, the baseline characteristics are similar to those in previous studies of people with dementia, supporting the reliability of the results in this group. This was a secondary analysis of the original trial and thus was not a randomized trial, although there were no significant differences in baseline characteristics between the intervention and control groups. Neuropsychiatric measures were secondary endpoints of the study, ${ }^{13}$ so NPS scores were low at baseline.
The results of this study are in line with those of the few previous studies examining the relationship between NPS and falls. ${ }^{8-10}$ Our study suggests that NPS are independent risk factors for falls. The fall risk related to NPS could be in part due to less day time physical activity because of apathy, the most common NPS. Low activity may in turn lead to frailty and risk of falls. ${ }^{25}$ This relationship should be more carefully evaluated in future studies, for example with activity monitors. The study intervention did not aim to reduce psychotropic use, and therefore it seems likely that the beneficial effects were related to exercise.

In accord with earlier studies, the number of NPS in our study was low. ${ }^{26}$ Our study was originally designed to improve physical functioning, but 175 participants scored at least 1 point on the NPI, and 38 scored 20 points or more. A clear linear relationship emerged between NPI score and falls in the control group.

As shown in our previous study, no significant changes in total NPI score or individual symptoms were detected in the intervention or control group. This may be because of the small number of NPS at baseline. ${ }^{13}$ Thus, the mechanism behind the interaction of exercise, NPS, and falls was due not to a decrease in NPS but probably to the beneficial effects of exercise on muscles and balance. Even though $70 \%$ the study population was at risk of malnutrition, they benefitted from the intervention. Another potential mechanism is improvement in executive functioning because of the exercise intervention reported in an earlier study examining the effects of exercise on cognition. ${ }^{27}$ NPS in AD are common. ${ }^{28}$ They increase caregiver burden and predict poor prognosis, including admission to institutional care. ${ }^{4}$ Although our intervention did not decrease the number of symptoms, it had an effect on the consequences of NPS, specifically falls.

\section{CONCLUSIONS}

This study revealed that NPS are linearly associated with number of falls and that exercise has a significant modifying effect on the risk of falling in community-dwelling people with AD and NPS. The findings suggest that exercise has the potential to reduce the risk of falls associated with NPS. Further studies are needed to confirm these results.

\section{ACKNOWLEDGMENTS}

Financial Disclosure: This work was supported by the Social Insurance Institution of Finland, the Central Union for the Welfare of the Aged, the Sohlberg Foundation, and the King Gustaf V and Queen Victoria Foundation.

Conflict of Interest: TS has had educational, consultative cooperation with several companies, including OrionPharma, Amgen, Novartis, Servier, Boehringer, MSD, Duodecim. Funding from IMI, Helsinki University Hospital, and Kela. MR has been investigator in many Alzheimer's drug development studies (Janssen-Cilag, Pfizer, Servier, Boehringer-Ingelheim, Biogen). She is a principal investigator in an aducanumab study (Biogen) in Helsinki, Finland. Shee has cooperated with patient and scientific associations, Kela, Duodecim, University of Helsinki. Grants over 5 years ago (biggest from Kela). Little amount 
of stocks from Orion. HÖ, H-MR has had minor educational cooperation with Astellas Pharma.

Author Contributions: Study concept and design: Kautiainen, Strandberg, Raivio, Laakkonen, Pitkälä. Acquisition, analysis, and interpretation of data: Roitto, Kautiainen, Öhman, Strandberg, Raivio, Laakkonen, Pitkälä. Preparation of paper and or critical revision for relevant intellectual content: Roitto, Kautiainen, Öhman, Strandberg, Raivio, Laakkonen, Pitkälä. Approval of final manuscript: Roitto, Kautiainen, Öhman, Strandberg, Raivio, Laakkonen, Pitkälä. Kaisu H. Pitkälä had full access to the data used in the study, is responsible for the integrity of the data and the accuracy of data analysis, and is the guarantor.

Sponsor's Role: The sponsors had no role in study design, subject recruitment, methods, data collection, analysis, interpretation of results, preparation of the paper, or in the decision to submit for publication. The authors were independent researchers not associated with the sponsors.

\section{REFERENCES}

1. McKhann GM, Knopman DS, Chertkow $\mathrm{H}$ et al. The diagnosis of dementia due to Alzheimer's disease: Recommendations from the National Institute on Aging-Alzheimer's Association workgroups on diagnostic guidelines for Alzheimer's disease. Alzheimers Dement 2011;7:263-269.

2. van Doorn C, Gruber-Baldini AL, Zimmerman S et al. Dementia as a risk factor for falls and fall injuries among nursing home residents. J Am Geriatr Soc 2003;51:1213-1218.

3. Nowrangi MA, Lyketsos CG, Rosenberg PB. Principles and management of neuropsychiatric symptoms in Alzheimer's dementia. Alzheimers Res Ther 2015;7:12.

4. Kales HC, Gitlin LN, Lyketsos CG. Assessment and management of behavioral and psychological symptoms of dementia. BMJ 2015;350:h369.

5. Welmerink DB, Longstreth WT, Lyles MF et al. Cognition and the risk of hospitalization for serious falls in the elderly: Results from the cardiovascular health study. J Gerontol A Biol Sci Med Sci 2010;65A:1242-1249.

6. Tinetti ME, Speechley M, Ginter SF. Risk factors for falls among elderly persons living in the community. N Engl J Med 1988;319:1701-1707.

7. van Dijk PT, Meulenberg OG, van de Sande HJ et al. Falls in dementia patients. Gerontologist 1993;33:200-204.

8. Hasegawa J, Kuzuya M, Iguchi A. Urinary incontinence and behavioral symptoms are independent risk factors for recurrent and injurious falls, respectively, among residents in long-term care facilities. Arch Gerontol Geriatr 2010;50:77-81.

9. Suzuki M, Kurata S, Yamamoto E et al. Impact of fall-related behaviors as risk factors for falls among the elderly patients with dementia in a geriatric facility in Japan. Am J Alzheimers Dis 2012;27:439-446.

10. Sylliaas H, Selbaek G, Bergland A. Do behavioral disturbances predict falls among nursing home residents? Aging Clin Exp Res 2012;24:251-256.
11. Burton E, Cavalheri V, Adams R et al. Effectiveness of exercise programs to reduce falls in older people with dementia living in the community: A systematic review and meta-analysis. Clin Interv Aging 2015;10:421-434.

12. Pitkälä KH, Pöysti MM, Laakkonen $M$ et al. Effects of the Finnish Alzheimer Disease Exercise Trial (FINALEX): A randomized controlled trial. JAMA Intern Med 2013;173:894-901.

13. Öhman H, Savikko N, Strandberg TE et al. Effects of exercise on neuropsychiatric symptoms and institutionalizations in FINALEX study. Eur Geriatr Med 2017;8:153-157.

14. Cummings JL. The Neuropsychiatric Inventory: Assessing psychopathology in dementia patients. Neurology 1997;48:S10-S16.

15. Folstein MF, Folstein SE, McHugh PR. 'Mini-mental state'. A practical method for grading the cognitive state of patients for the clinician. J Psychiatr Res 1975;12:189-198.

16. Hughes CP, Berg L, Danziger WL et al. A new clinical scale for the staging of dementia. Br J Psychiatry 1982;140:566-572.

17. Pollak N, Rheault W, Stoecker JL. Reliability and validity of the FIM for persons aged 80 years and above from a multilevel continuing care retirement community. Arch Phys Med Rehabil 1996;77:1056-1061.

18. Guralnik JM, Simonsick EM, Ferrucci L et al. A short physical performance battery assessing lower extremity function: Association with self-reported disability and prediction of mortality and nursing home admission. J Gerontol 1994;49:M85-M94.

19. Guigoz Y, Lauque S, Vellas BJ. Identifying the elderly at risk for malnutrition. The Mini Nutritional Assessment. Clin Geriatr Med 2002;18:737-757.

20. Charlson ME, Pompei P, Ales KL et al. A new method of classifying prognostic comorbidity in longitudinal studies: Development and validation. J Chronic Dis 1987;40:373-383.

21. Aalten P, Verhey FRJ, Boziki M et al. Neuropsychiatric syndromes in dementia. Dement Geriatr Cogn Disord 2007;24:457-463.

22. Alexopoulos GS, Abrams RC, Young RC et al. Cornell Scale for depression in dementia. Biol Psychiatry 1988;23:271-284.

23. Pitkälä KH, Raivio MM, Laakkonen ML et al. Exercise rehabilitation on home-dwelling patients with Alzheimer's disease-a randomized, controlled trial. Study protocol. Trials 2010;11:92.

24. Hannan MT, Gagnon MM, Aneja J et al. Optimizing the tracking of falls in studies of older participants: Comparison of quarterly telephone recall with monthly falls calendars in the MOBILIZE Boston study. Am J Epidemiol 2010;171:1031-1036.

25. David R, Mulin E, Friedman L et al. Decreased daytime motor activity associated with apathy in Alzheimer disease: An actigraphic study. Am J Geriatr Psychiatry 2012;20:806-814.

26. Telenius EW, Engedal K, Bergland A. Effect of a high-intensity exercise program on physical function and mental health in nursing home residents with dementia: An assessor blinded randomized controlled trial. PLoS One 2015; 10:e0126102.

27. Öhman H, Savikko N, Strandberg TE et al. Effects of exercise on cognition: The Finnish Alzheimer disease exercise trial: A randomized, controlled trial. J Am Geriatr Soc 2016;64:731-738.

28. Lyketsos CG, Lopez O, Jones B et al. Prevalence of neuropsychiatric symptoms in dementia and mild cognitive impairment: Results from the cardiovascular health study. JAMA 2002;288:1475-1483. 